\title{
Temperature dependence of positronium decay rates in gases
}

\author{
R S Vallery $\dagger$, A E Leanhardt†, M Skalsey $\dagger$ and D W Gidley $\dagger$ \\ $\uparrow$ Randall Laboratory of Physics, University of Michigan, 500 E University Ave, Ann Arbor, \\ MI 48109, USA \\ \$ Department of Physics, Massachusetts Institute of Technology, 77 Massachusetts Ave, \\ Cambridge, MA 02139 USA \\ E-mail: vallery@umich.edu
}

Received 1 September 1999, in final form 5 January 2000

\begin{abstract}
The decay rate of orthopositronium (o-Ps) formed and thermalized in eight different gases is systematically investigated as a function of temperature. The o-Ps collisional quenching rate is observed to increase with temperature, $T$, for $\mathrm{He}, \mathrm{Ne}, \mathrm{Ar}, \mathrm{N}_{2}$, ethane, methane, isobutane and neopentane. All of the gases except ethane and methane increase linearly over the investigated temperature range, $300 \mathrm{~K}<T<600 \mathrm{~K}$. Recent theoretical work for noble gases at elevated temperatures suggests a linear increase in the collisional quenching rate with temperature at the low densities used herein where collective phenomena such as bubble formation and density fluctuations are not present. When comparisons can be made with previous experimental results the agreement is generally poor. Precision o-Ps decay rate measurements using gases are also discussed concerning systematic effects due to the energy dependence of the collisional quenching rate.
\end{abstract}

\section{Introduction}

Positronium is the short-lived hydrogen-like bound state of an electron and its antiparticle, a positron. The ground state consists of a triplet of spin-1 states, orthopositronium (o-Ps), and a single spin-0 state, parapositronium (p-Ps). Positronium formation and decay has been investigated for a wide range of solids, liquids and gases. In particular, collisions between normal gas atoms and the exotic atom of positronium from its initial formation energy of typically a few $\mathrm{eV}$ to thermal energies are interesting if only for the wide variety of processes that can occur [1]. The two most unique processes are related to the very light mass of neutral Ps relative to its target gas atoms and to the fact that Ps is a particle-antiparticle system that annihilates. The low mass, and hence long de Broglie wavelength, not only renders lowenergy collisions with atoms inherently quantum mechanical [2,3], but it also results in very long Ps thermalization times if elastic scattering is the dominant scattering process for energy loss. Ps thermalization rates and the related momentum transfer cross sections for gases are challenging to predict and measure; and, in fact, our recently measured cross sections in $\mathrm{He}$ and $\mathrm{H}_{2}$ [4] are nearly an order of magnitude below the then existing theoretical predictions. The experimental thermalization results are not in good agreement either [5]. The annihilation processes of Ps have been studied extensively versus gas composition and pressure [6]. The annihilation processes include self-annihilation as well as collisional quenching of the positron in Ps by annihilation with molecular/atomic electrons. Any decay rate study must ensure that Ps is thermalized or account taken of the velocity dependence of collisional annihilation [2]. 
The velocity/temperature dependence of collisional quenching has become crucial to interpreting precision Ps decay rate spectra in low and medium gas densities since it is now known [4] that Ps has not always been completely thermalized in these measurements and hence, the collisional quenching rate might be changing during the Ps decay rate measurement interval. This is particularly true in the 1989 precision measurement of the Ps triplet decay rate [7], wherein the decay rate was measured at low gas densities and the vacuum value of the decay rate determined as a test of QED theory by extrapolation to zero density. The measured decay rate was significantly higher than existing theory [8] and a more recent measurement in silica powders [9]. It is thus important to determine whether this difference can be attributed to incomplete Ps thermalization.

Unfortunately, prior to this investigation, there were relatively little data on the decay rate temperature dependence and what did exist were often acquired in the wrong temperature range for our purposes, or at an inappropriately high gas density. In some cases the data simply appear to be contradictory. Our goal in this paper is to present systematically measured temperature-dependent collisional Ps annihilation rates in a temperature range above $295 \mathrm{~K}$ and for a variety of gases that specifically include those used in the 1989 precision decay rate measurement [7].

\section{Quenching studies}

A positronium decay rate experiment generates an annihilation time spectrum by measuring the time interval between the emission of a positron and the eventual decay of Ps. The time required for the positron to thermalize and form Ps is of the order of $1 \mathrm{ps}$ at the gas densities used in this study and is negligible compared to the measured Ps lifetime. The time spectrum of the exponential decay is analysed to extract a Ps decay rate, $\lambda(n, T)$ (or lifetime, $\tau=1 / \lambda$ ) for the Ps starting at a particular time after formation. In general, $\lambda$ depends on the gas number density, $n$, and temperature, $T$. In gases, beta-decay positrons are slowed down until it is energetically favourable to form Ps $(\approx 10 \mathrm{eV})$. The formation of Ps is known to occur up to about $100 \mathrm{eV}$ in rarified gases [1]. In denser media, if Ps has a kinetic energy greater than its binding energy of $6.8 \mathrm{eV}$, there is a high probability of collisional dissociation [4]. After formation, $\mathrm{p}$-Ps decays rapidly ( $\tau=125 \mathrm{ps}$ ) into two photons, while unperturbed o-Ps can live up to $142 \mathrm{~ns}$ in vacuum and decays into three photons. Formed at a typical average of a few eV, o-Ps undergoes numerous collisions with the gas atoms as it thermalizes. When o-Ps scatters with gas atoms it may be rapidly annihilated in the collision. The annihilation process occurs when the positron in o-Ps overlaps with an atomic electron of the opposite spin, resulting in rapid singlet state annihilation, comparable to p-Ps. The effect of this singlet collisional quenching is to increase the observed decay rate, $\lambda(n, T)$, above the vacuum decay rate, $\lambda_{T}$, as given by

$$
\lambda(n, T)=\lambda_{T}+\lambda_{\mathrm{q}}(n, T)
$$

where $\lambda_{\mathrm{q}}(n, T)$ is the density- and temperature-dependent collisional quenching rate. Ignoring three-body interactions, the collisional quenching rate is, from gas kinematics,

$$
\lambda_{\mathrm{q}}(n, T)=n \sigma(v) v
$$

where $\sigma(v)$ is the quenching cross section and $v$ is the velocity of the o-Ps. Therefore, $\lambda_{\mathrm{q}}(n, T)$ depends on the temperature of the gas once o-Ps is thermalized. Notice that the relatively slow motion of the gas atoms can be ignored. If the product $\sigma(v) v$ depends on temperature, the measured decay rate will then change as o-Ps thermalizes. Since o-Ps can have long thermalization times [4], it cannot necessarily be assumed that the o-Ps is 
completely thermalized at the time for which $\lambda(n, T)$ is determined. Therefore, it is necessary to determine the dependence of $\sigma(v) v$ on velocity in order to completely understand the impact of thermalizing o-Ps on some gas decay rate experiments. The impact of thermalizing o-Ps (which was thought to be sufficiently understood) can be clearly seen in figure 5 of the 1989 decay rate measurement [7] by the asymptotic decrease of the fitted value of $\lambda(n, T)$ as a function of the start time of the fit to a constant value.

The pertinent processes necessary to understand the nature of the Ps decay rate and quenching as a function of temperature (or Ps velocity) were outlined and estimated several decades ago [3]. Due to the complicated nature of the interaction, the energy dependence of quenching has only been recently investigated more extensively theoretically [2]. If Ps is at a sufficiently low velocity, it undergoes purely s-wave scattering with the gas and $\sigma(v)$ is inversely proportional to the velocity and hence $\lambda_{\mathrm{q}}(n, T)$ is independent of $v$. This result was confirmed theoretically [2] for sufficiently low gas temperatures. Unfortunately, it was not made clear in [2] at what temperature the quenching rate should begin to deviate from the temperature-independent behaviour. Also, the exact size of the increase over the modest temperature range used in this experiment has yet to be calculated. Moreover, the calculations for $\lambda_{q}(n, T)$ have only been made for xenon over a limited number of temperatures and have yet to be calculated for other noble gases. Also, it is unknown whether these calculations are applicable to the more complicated molecular gases.

The experimental situation for the temperature dependence of $\lambda_{\mathrm{q}}(n, T)$ is similarly inconclusive. Several experiments in a variety of gases have been performed which measure the value of the quenching rate over a range of temperatures. The results and conditions of these experiments varied significantly. It is reported that for ammonia [10], nitrogen [11], methane [12], $\mathrm{H}_{2}$ [13], $\mathrm{Ne}$ [14], $\mathrm{Ar}$ [14], $\mathrm{Xe}$ [15] and $\mathrm{He}$ [16], the decay rate is independent of temperature. However, a separate experiment in He [17] indicates an increasing decay rate and another measurement in ethane [18] indicates that the decay rate decreases with temperature. Most of the experiments were conducted at either cryogenic temperatures and/or very high densities in order to observe exotic effects of positronium decay such as 'bubble' formation or density fluctuations. Moreover, the temperature range within each particular investigation was usually limited, making it difficult to determine the exact nature of any temperature variation. It is therefore difficult to make any precise determination of $\lambda_{\mathrm{q}}(n, T)$ just above room temperature on the basis of previous experiments. Given the lack of systematically acquired data and the difficulty in using existing theoretical calculations to make precise predictions for the temperature dependence of $\lambda_{\mathrm{q}}$, it is necessary to investigate the quenching decay rate experimentally near and above room temperature and at pressures high enough to thermalize Ps, but low enough where three-body collisions are not a concern.

\section{Experimental procedure}

A schematic of the present apparatus is shown in figure 1. A standard timing spectrometer measures the decay rate of positronium in a fixed density of gas over the temperature range of interest. Positronium is formed in various densities of research grade gas in a $50 \mathrm{~cm}^{3}$ high-pressure stainless steel gas cell that can be isolated from the gas handling system in order to maintain a constant density while heating. The positron source is $15 \mu \mathrm{Ci}{ }^{22} \mathrm{Na}$ acetate deposited as a water solution and dried on, and then sealed between thin $(2.5 \mu \mathrm{m})$ nickel foils. The source is mounted in the centre of the gas cell in order to minimize the number of positrons that annihilate in the cell walls. The gas cell is wrapped in heating tapes and encased in an insulated aluminium box in order to maintain a reasonably uniform temperature across the cell and isolating valve. A constant temperature is maintained using a temperature 


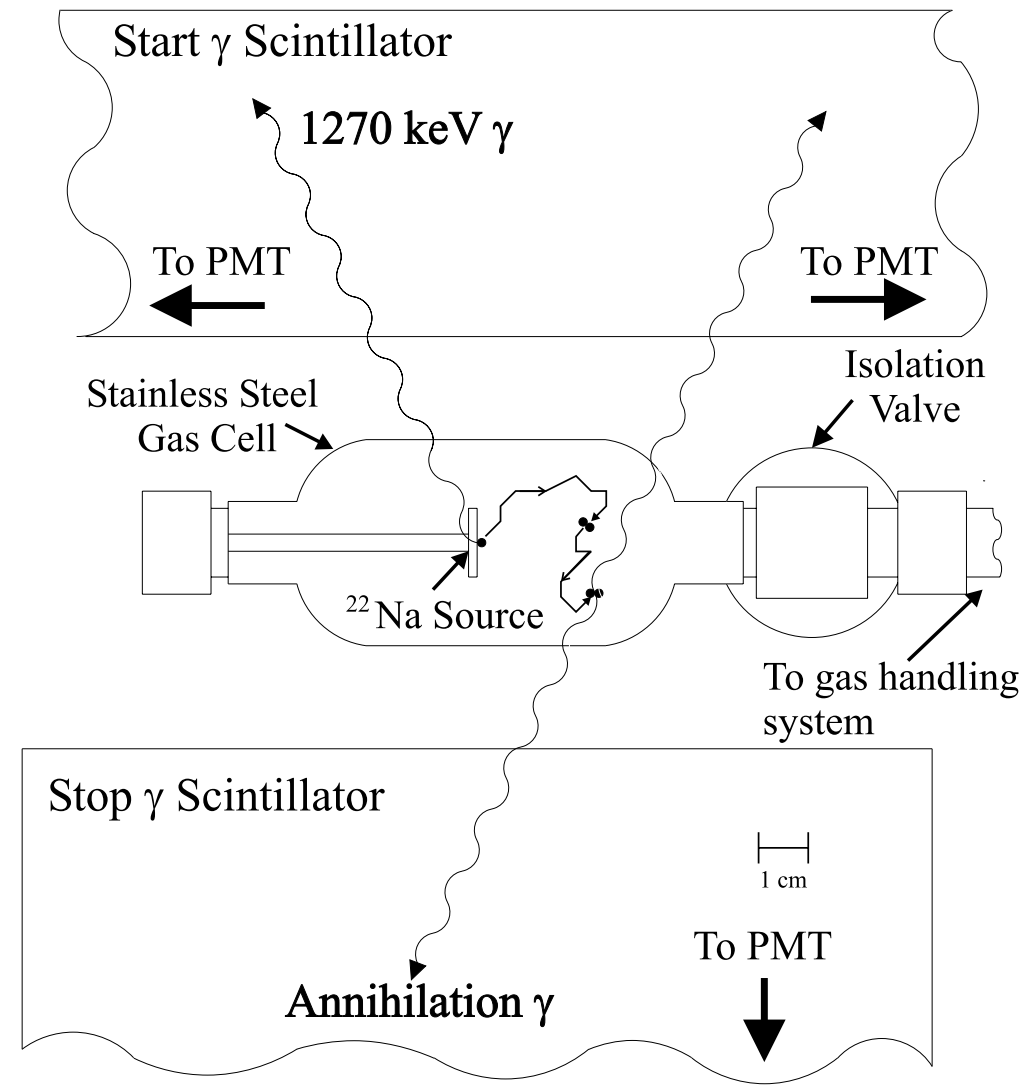

Figure 1. Schematic of the apparatus. A heatable stainless steel gas cell, which can be isolated from the gas handling system, is used to maintain a constant density of gas over a temperature range of $300-600 \mathrm{~K}$. A ${ }^{22} \mathrm{Na}$ source is inserted in the centre of the cell to supply positrons. Two plastic scintillators connected to phototubes (PMT) are used to acquire start and stop signals for the timing system. Not shown are heaters, insulation and thermocouples.

controller to cycle power to the heating tapes and using a thermocouple mounted on the centre of the cell as a reference. The effective range of the cell is $273-600 \mathrm{~K}$ and it is stable to $\pm 1 \mathrm{~K}$. The temperature is also monitored at several other locations along the cell and the value quoted is the average of the temperature profile of the main cell, covering $95 \%$ of the total volume. The temperature along the cell varies by at most $10 \mathrm{~K}$ across the majority of the cell and by up to $50 \mathrm{~K}$ at the valve when the experiment is at the maximum operating temperature.

The lifetime spectra of Ps decaying in a gas are acquired using a timing spectrometer with a time resolution of $2.5 \mathrm{~ns}$ FWHM. Two fast plastic scintillators coupled to photomultiplier tubes are placed on opposite sides of the gas cell and serve as start and stop detectors. The start signal is derived from the $1270 \mathrm{keV}$ nuclear $\gamma$-ray that is emitted concomitantly with the positron from the ${ }^{22} \mathrm{Na}$ source. The stop signal is derived from any one of the two or three annihilation $\gamma$-rays. The phototube signals are amplified and then discriminated (using constant fraction discriminators) and the lifetime for each event is determined with a CAMAC Lecroy 4204 time-to-digital converter. The resulting lifetime histogram is stored for later analysis and typically contains $12-48 \mathrm{~h}$ of data. Spectra are acquired at increasing temperatures in the range 
$20-300{ }^{\circ} \mathrm{C}$ and at the same points while cycling back down to $20^{\circ} \mathrm{C}$ to ensure reversibility and reproducibility. The constancy of the decay rate measured at room temperature during the course of temperature cycling is used as a monitor for any leakage or contamination of the cell's contents.

The gas cell is prepared for a temperature scan by evacuating it with a mechanical pump. The system is then filled to $3-5 \mathrm{~atm}$ of the gas to be used and pumped out. This flushing is repeated several times to ensure that the cell is free from contamination. For $\mathrm{N}_{2}, \mathrm{Ar}$ and $\mathrm{Ne}$, the cell is filled to the desired pressure, as measured by a regulator on the gas cylinder, and then the cell is isolated from the rest of the gas handling system. Neopentane, isobutane and ethane, which have low liquefaction pressures, are allowed to fill the gas handling system and the gas cell to their maximum pressure, 1.5, 3.1 and $38 \mathrm{~atm}$, respectively. Methane was allowed to fill the gas cell to its cylinder pressure of $111 \mathrm{~atm}$. Some of the gas is then pumped out before sealing to ensure that no condensation occurred in the cell that could change the gas density as the temperature is increased. All spectra acquired with $\mathrm{He}$, and some with $\mathrm{Ne}$, are taken with a small admixture of either neopentane or isobutane and will be presented in more detail later. In all cases, the final gas pressure is sufficiently high so as to thermalize the positronium rapidly and thus thermalization effects are not a concern.

\section{Data analysis}

Once the lifetime spectrum at a particular temperature has been acquired, $\lambda(n, T)$ is determined by fitting it to one or two exponential decay rates and a background using a maximum-likelihood $\chi^{2}$-minimization routine. A typical spectrum has several prominent features which include: (a) a prompt peak that is due to directly annihilating positrons and parapositronium (lifetime of $125 \mathrm{ps}$ ); (b) a short-lived exponential component due to slow positrons, i.e. positrons that have fallen below the Ps formation threshold and must annihilate directly with an electron (see [19] for more details); and (c) a long-lived exponential component of collisionally quenched o-Ps. In $\mathrm{N}_{2}, \mathrm{Ar}$ and $\mathrm{Ne}$ gas it is necessary to fit to two decay rates since the slow positron component does not decay fast enough at modest gas density and appears beyond the prompt peak. However, isobutane and neopentane are known to annihilate these slow positrons so rapidly that this component is fully quenched before the start time of the fitting program. Taking advantage of this property, all of the He data and some of the Ne data are taken with a small (30-150 Torr) admixture of isobutane or neopentane. In these cases it is only necessary to fit to a single o-Ps decay rate. The effect of the hydrocarbon mix-gas on $\lambda_{\mathrm{q}}(n, T)$ and its temperature dependence is $1-10 \%$ and is straightforwardly accounted for in the analysis. In all cases the start time of the fit is progressively stepped out and the quenched orthopositronium decay rate, $\lambda_{T}$, is chosen in a region where the successively fitted values are statistically consistent, usually at $t \cong 80-100 \mathrm{~ns}$. This ensures that Ps is thermalized in the gas and there are no extraneous lifetime components arising in the spectrum.

Once the fitted value of $\lambda(n, T)$ is determined, the quenching rate, $\lambda_{\mathrm{q}}(n, T)$, is calculated from equation (1) using a nominal value of $\lambda_{T}=7.045 \pm 0.005 \mu \mathrm{s}^{-1}$ for the vacuum decay rate $[7,9]$. While a filling of the gas cell has a single density, multiple fillings of a gas are taken at different densities which are known to no better than a few per cent. The simple linear density dependence of the quenching rate is removed by normalizing $\lambda_{\mathrm{q}}(n, T)$ to the room temperature value of $\lambda_{\mathrm{q}}(n, 295 \mathrm{~K})$ for each gas filling. The resulting density-normalized quenching rate, $\Lambda_{\mathrm{q}}(T)$ is given by

$$
\Lambda_{\mathrm{q}}(T)=\frac{\lambda_{\mathrm{q}}(n, T)}{\lambda_{\mathrm{q}}(n, 295 \mathrm{~K})}=\frac{\sigma(v(T)) v(T)}{\sigma(v(295 \mathrm{~K})) v(295 \mathrm{~K})} .
$$


$\Lambda_{\mathrm{q}}(T)$ parametrizes the relative change of $\sigma(v) v$ with temperature. $\Lambda_{\mathrm{q}}(T)$ is corrected for two systematic effects that reduce the gas density at elevated temperature. The first correction takes into account the simple volume expansion of the stainless steel cell which requires an increase $\left(55 \pm 5 \mathrm{ppm}{ }^{\circ} \mathrm{C}^{-1}\right)$ in the fitted value of $\Lambda_{\mathrm{q}}(T)$ (pressure-induced expansion of the gas cell is negligible). The second correction of $12 \pm 10 \mathrm{ppm}^{\circ} \mathrm{C}^{-1}$ arises from a temperature difference (typically $50 \mathrm{~K}$ at $600 \mathrm{~K}$ ) between the main gas cell and a small volume of gas in the isolation valve that produces a slight decrease in the density in the main gas cell. Hence, it is necessary to increase the measured value of $\Lambda_{\mathrm{q}}(T)$ by a combined correction of $+67 \pm 11 \mathrm{ppm}^{\circ} \mathrm{C}^{-1}$.

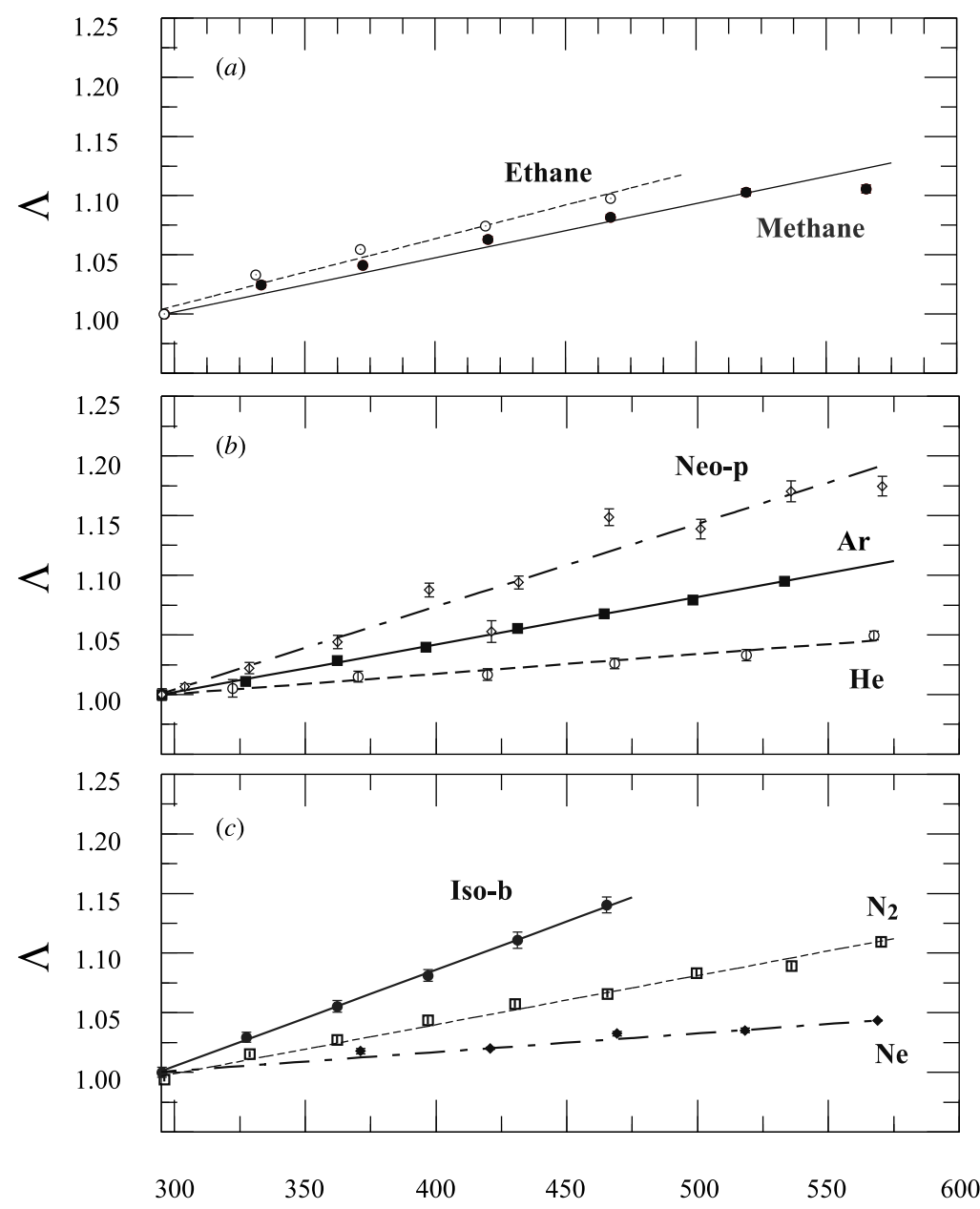

\section{Temperature (K)}

Figure 2. The value of $\Lambda$, the normalized quenching rate of orthopositronium, as a function of temperature for $\mathrm{Ne}, \mathrm{N}_{2}, \mathrm{He}, \mathrm{Ar}$, ethane, methane, isobutane (Iso-b) and neopentane (Neo-p). The lines shown are linear fits to the data and the size of the increase per degree, $\alpha$, is presented in table 1. For data where no error bars are evident, the error bars are smaller than the plotting symbol. 


\section{Discussion}

The corrected values of the normalized decay rate, $\Lambda_{\mathrm{q}}(T)$, for all of the gases used in this experiment are plotted as a function of temperature in figure 2. One clear feature in all these data is that $\Lambda_{\mathrm{q}}(T)$ increases with temperature in contrast to the previous constant results for ethane [18], methane [12], neon [14], argon [13], nitrogen [11] and helium [16], but in nominal agreement with the second helium result [17]. Since most of these previous data were taken at temperatures of less than $300 \mathrm{~K}$, direct comparison of quenching results obtained over different temperature ranges may not be appropriate. It is likely that s-wave scattering is the predominant mode at low $T$, whereas in the temperature range of this experiment, the energy of the positronium may be sufficiently high so that higher partial waves begin to contribute significantly.

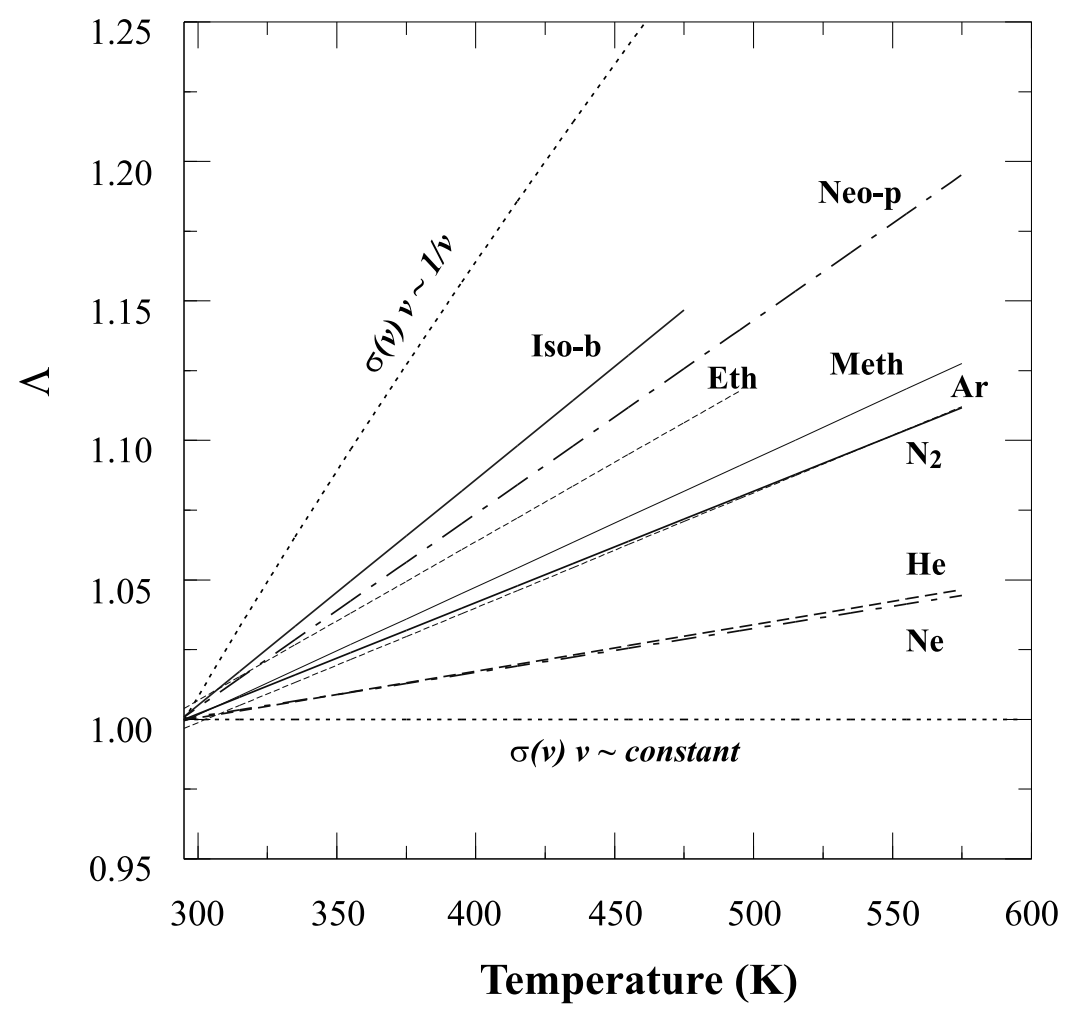

Figure 3. The linear fits from figure 2 are plotted to show the increase in $\Lambda$ for all of the gases.

To quantify the temperature dependence in $\Lambda_{\mathrm{q}}(T)$ for each gas the data in figure 2 are fitted to a straight line (higher-order polynomial fitting is not required in any of the gases except ethane and methane). The fits are shown in figure 2 with the data and collectively in figure 3 . In addition, two curves have been included in figure 3 to guide the eye. The horizontal line is for $\sigma(v) \sim 1 / v$ resulting in $\Lambda_{\mathrm{q}}(T) \sim$ constant, while the second curve is for $\sigma(v) \sim$ constant and hence, $\Lambda_{\mathrm{q}}(T) \sim v \sim \sqrt{T / 295 \mathrm{~K}}$. The slope parameter $\alpha$, the fractional increase of $\Lambda_{\mathrm{q}}(T)$ in $\mathrm{ppm}^{\circ} \mathrm{C}^{-1}$ is given in table 1 for those gases in which linear fits in temperature are appropriate. The error in the temperature correction factor of $+67 \pm 11 \mathrm{ppm}^{\circ} \mathrm{C}^{-1}$ has an $11 \mathrm{ppm}$ systematic effect on $\alpha$. The $0.005 \mu \mathrm{s}^{-1}$ systematic uncertainty in the nominal value of $\lambda_{T}$ has a negligible effect on the determination of $\alpha$. It is clear from figures $2(b)$ and $(c)$ 
Table 1. Temperature dependence of Ps quenching in gases. The pressure of the gas, the size of the increase in quenching, $\alpha$, and the $\chi^{2}$ of the fit are presented. The value of $\alpha$ is found by fitting the normalized decay rates as linear in temperature. The methane and ethane results are clearly nonlinear and the $\alpha$ values quoted are for the fitted data shown in figures 2 and 3 .

\begin{tabular}{llll}
\hline Gas & Pressure $(\mathrm{psig})$ & $\alpha\left(\mathrm{ppm}{ }^{\circ} \mathrm{C}^{-1}\right)$ & $\chi^{2} / \mathrm{DF}$ \\
\hline $\mathrm{He}$ & 350 & $167 \pm 17$ & $3.4 / 5$ \\
$\mathrm{Ne}$ & $250-400$ & $158 \pm 11$ & $12 / 4$ \\
$\mathrm{Ar}$ & $250-350$ & $338 \pm 12$ & $10 / 11$ \\
$\mathrm{~N}_{2}$ & $150-360$ & $412 \pm 15$ & $18 / 7$ \\
Isobutane & $15-35$ & $810 \pm 39$ & $1 / 4$ \\
Neopentane & 7 & $694 \pm 23$ & $46 / 9$ \\
Methane & $200-300$ & $458 \pm 13$ & $50 / 5$ \\
Ethane & 160 & $569 \pm 26$ & $1.8 \times 10^{6} / 3$ \\
\hline
\end{tabular}

that the noble gases, nitrogen, neopentane and isobutane increase linearly in temperature. The distribution of neopentane data in figure $2(b)$ is statistically anomalous, but does not point to a nonlinear fit in $\Lambda_{\mathrm{q}}(T)$. While linear fits are included for methane and ethane, it can be seen in figure 2( $a$ ) that they deviate systematically from linearity and both tend to drop below the linear fit. The noble gas results from this measurement appear to be consistent with the predicted trend in [2] that $\alpha$ increases proportionally to the cross section of the atom. However, to investigate the details of the temperature dependence, more detailed calculations are needed for each gas.

The situation for the more complicated molecular gases is interesting. Neopentane and isobutane quenching increases linearly with temperature, while ethane and methane quenching deviates strongly from linearity when plotted against temperature or velocity. It should be noted that for isobutane and ethane there are no data above $473 \mathrm{~K}$. At this temperature the decay rate drops by several standard deviations and, when the decay rate is remeasured at room temperature, the value deviates significantly from the previously measured value. Only data below this temperature are reproducible and hence, reliable. The increase in temperature in the temperature range investigated is largest for neopentane and isobutane, while it is smaller for ethane and methane.

\section{Conclusions}

The temperature dependence of the o-Ps quenching rate has been measured above room temperature in several gases at low to medium densities. All of these gases present a modest, but clear, increase in the quenching rate. With the exception of ethane and methane, this increase is linear with $T$ from 300 to $600 \mathrm{~K}$, in qualitative agreement with a more complete theory [2]. Our results are not consistent with the temperature-independent trends established in some of the gases below room temperature. It is possible that this difference observed in the two temperature regimes may simply be a manifestation of the complete domination of s-wave scattering at low Ps energies. However, it seems reasonable to expect that some vestige of our observed linear dependence on $T$ would continue below room temperature. It would thus be interesting to have more precise and systematic measurements below $300 \mathrm{~K}$. Further calculations throughout these experimentally accessible temperature regimes are certainly warranted as well.

Given that there is an energy/temperature dependence for the decay rate and that Ps thermalization times are significantly longer than previously expected [4], it is then necessary to 
consider the impact of thermalizing Ps (and hence a time-dependent decay rate) on any lifetime measurement in a gas. If Ps has not completely thermalized in a particular measurement, incorrect conclusions as to the density, velocity or time dependence of the decay rate may be made. This is especially important in experiments in which an extrapolation of $\lambda(n)$ is made to zero density, such as the 1989 precision measurement of the vacuum decay rate [7] which used low densities of neon, nitrogen, isobutane and neopentane as the formation media. Our measurements reported herein of the increase in $\lambda_{\mathrm{q}}(n, T)$ with temperature for these particular gases, coupled with the measured long thermalization times [4] for those same gases, allow one to correct the extrapolated values of $\lambda_{T}$. Such corrections are being determined for each gas used in the 1989 experiment. Complete details will be reported elsewhere.

\section{Acknowledgments}

We would like to thank R S Conti, G W Ford, R R Lewis, Phil Shaltis and J J Engbrecht for their insights into this work. This research is funded by National Science Foundation Grant PHY-9731861 and the University of Michigan.

\section{References}

[1] Laricchia G 1996 Hyperfine Interact. 10071

[2] Miller B N, Reese T L and Worrel G A 1996 Can. J. Phys. 74548

[3] Massey H S W and Mohr C B O 1954 Proc. Phys. Soc. A 67695

[4] Skalsey M, Bithell R K, Engbrecht J J, Vallery R S and Gidley D W 1998 Phys. Rev. Lett. 803727

[5] Nagashima Y et al 1995 Phys. Rev. A 52258

[6] Coleman P G, Griffith T C, Heyland G R and Killeen T L 1975 J. Phys. B: At. Mol. Phys. 81734

[7] Westbrook C I, Gidley D W, Conti R S and Rich A 1989 Phys. Rev. A 405489

[8] Adkins G S 1996 Phys. Rev. Lett. 764903

[9] Asai S, Orito S and Shinohara N 1995 Phys. Lett. B 357475

[10] McNutt J D, Kinnison W W and Ray A D 1974 J. Chem. Phys. 604730

[11] Kawaranti T, Nakayama Y and Mizogawa T 1988 Phys. Rev. A 372855

[12] McNutt J D, Summerour V B, Ray A D and Killeen T L 1975 J. Chem. Phys. 621777

[13] McNutt J D, Sharma S C, Franklin H M and Woodall M A II 1979 Phys. Rev. A 20357 Laricchia G, Charlton M, Beling C D and Griffith T C 1987 J. Phys. B: At. Mol. Phys. 201865

[14] Canter K F and Roellig L O 1975 Phys. Rev. A 12386

[15] Tuomissari M, Rytsölä K and Hautojärvi P 1988 J. Phys. B: At. Mol. Opt. Phys. 213917

[16] Fox R A, Canter K F and Fishbein M 1977 Phys. Rev. A 151340

[17] Canter K F, McNutt J D and Roellig L O 1975 Phys. Rev. A 12375

[18] Sharma S C, McNutt J D, Eftekhari A and Heji R A 1981 J. Chem. Phys. 751226

[19] Griffith T C and Heyland G R 1978 Phys. Rep. 39169 